\title{
Exploring the potential of neutron imaging for life sciences On IMAT
}

\begin{tabular}{|c|c|}
\hline Journal: & Journal of Microscopy \\
\hline Manuscript ID & JMI-2018-0023.R1 \\
\hline Wiley - Manuscript type: & Themed Issue Paper \\
\hline Date Submitted by the Author: & 13-Sep-2018 \\
\hline Complete List of Authors: & $\begin{array}{l}\text { Burca, Genoveva; STFC, Rutherford Appleton Laboratory, ISIS Facility } \\
\text { Nagella, Srikanth; STFC, Rutherford Appleton Laboratory, Scientific } \\
\text { Computing Department } \\
\text { Clark, Thomas; University of Southampton Faculty of Engineering and the } \\
\text { Environment, Bioengineering Sciences Research Group; STFC, Rutherford } \\
\text { Appleton Laboratory, ISIS Facility } \\
\text { Tasev, Dimitar; Aberystwyth University, Department of Computer Science } \\
\text { Rahman, Imran; University of Oxford, Oxford University Museum of Natural } \\
\text { History } \\
\text { Garwood, Russell; School of Earth and Environmental Sciences, The } \\
\text { University of Manchester, UK ; Department of Earth Sciences, The Natural } \\
\text { History Museum London } \\
\text { Spencer, Alan; The Natural History Museum London, Department of Earth } \\
\text { Sciences; Imperial College London Department of Earth Science and } \\
\text { Engineering } \\
\text { Turner, Martin; University of Manchester, School of Computing Science; } \\
\text { STFC, Rutherford Appleton Laboratory } \\
\text { Kelleher, Joe; STFC, Rutherford Appleton Laboratory, ISIS Facility }\end{array}$ \\
\hline Keywords: & $\begin{array}{l}\text { neutron imaging, neutron computed tomography, neutron radiography, life } \\
\text { sciences, palaeontology, biology }\end{array}$ \\
\hline
\end{tabular}




\title{
Exploring the potential of neutron imaging for life sciences on IMAT
}

\author{
Genoveva Burca ${ }^{1, *}$, Sri Nagella ${ }^{2}$, \\ Thomas Clark ${ }^{1,3}$, Dimitar Tasev $^{4}$ \\ Imran A. Rahman ${ }^{5}$, Russell J. Garwood ${ }^{6,7}$, Alan R.T. Spencer ${ }^{7,8}$, \\ Martin J. Turner ${ }^{2,9}$, Joe F. Kelleher ${ }^{1}$
}

${ }^{1}$ STFC, Rutherford Appleton Laboratory, ISIS Facility, Harwell, OX11 0QX, UK

${ }^{2}$ Scientific Computing Department, Rutherford Appleton Laboratory, STFC, Harwell, OX11 OQX, UK

${ }^{3}$ Bioengineering Sciences Research Group, Faculty of Engineering and the Environment, University of Southampton, Southampton, SO17 1BJ, UK

${ }^{4}$ Department of Computer Science, Aberystwyth University, SY23 3DB, UK

${ }^{5}$ Oxford University Museum of Natural History, University of Oxford, OX1 3PW, UK

${ }^{6}$ School of Earth and Environmental Sciences, The University of Manchester, UK

${ }^{7}$ Department of Earth Sciences, The Natural History Museum London, London, UK

${ }^{8}$ Department of Earth Science and Engineering, Imperial College London, London, UK

${ }^{9}$ School of Computer Science, The University of Manchester, UK

Correspondence to: Genoveva Burca, STFC, Rutherford Appleton Laboratory, ISIS Facility, Harwell, OX11 0QX, UK. Tel +(44) 1235 446588; e-mail: Genoveva.Burca@stfc.ac.uk

Key words: neutron imaging, neutron computed tomography, neutron radiography, life sciences, palaeontology, biology 


\section{ABSTRACT}

Neutron imaging has been employed in life sciences in recent years and has proven to be a viable technique for studying internal features without compromising integrity and internal structure of samples in addition to being complementary to other methods such as Xray or magnetic resonance imaging. Within the last decade, a neutron imaging beamline, IMAT, was designed and built at the ISIS Neutron and Muon Source, UK, to meet the increasing demand for neutron imaging applications in various fields spanning from materials engineering to biology. In this paper, we present the first neutron imaging experiments on different biological samples during the scientific commissioning of the IMAT beamline mainly intended to explore the beamline's capabilities and its potential as a non-invasive investigation tool in fields such as agriculture (soil-plants systems), palaeontology and dentistry.

\section{1. INTRODUCTION}

2 Neutrons form a highly penetrating radiation that passes through matter without damaging or 3 structurally modifying it. This property makes neutrons an ideal tool for many kinds of

4 complementary material investigations. Moreover, the strong interaction of neutrons with 5 hydrogen, and their ability to distinguish between hydrogen and deuterium with no radiation 6 damage, makes neutrons a good probe for imaging biological specimens. Among the 7 experimental neutron techniques being developed in biology, one could mention: neutron 8 activation analysis related to the capture of neutrons, which is applied for isotope and element 9 analysis (Yunus et al., 2010); small-angle neutron scattering (Teixeira et al., 2008) used to understand the interaction between the complex molecular systems that make up living cells;

11 and neutron imaging (Anderson, McGreevy \& Bilheux, 2009) based on the capture and 
scattering of neutrons which provides information about the inner structure and, indirectly, about the composition of a sample due to the fact that different materials attenuate neutrons to a greater or lesser extent. While small-angle neutron scattering is well exploited in life sciences research, neutron imaging has become more appealing in the recent years through development and improvement of both the sources and the detector systems.

\section{NEUTRON IMAGING FACILITY IN UK, IMAT BEAMLINE}

The neutron imaging facilities, e.g. CONRAD at BER-II (Kardjilov et al., 2016), ANTARES at FRM-II (Heinz Maier-Leibnitz Zentrum et al., 2015), NEUTRA (Lehmann et al., 2001) and ICON (Kaestner et al., 2011) at PSI, RADEN at J-PARC (Kiyanagi et al., 2013), CG-1D at Oak Ridge Laboratory (Santodonato et al., 2015), have to serve the demands of an increasing number of users worldwide whether they are under operation at research reactors or spallation sources.

IMAT (Imaging and Materials Science \& Engineering) is the first neutron imaging and diffraction beamline at the ISIS Pulsed Neutron and Muon Source, based at Rutherford Appleton Laboratory (RAL) in the UK. Design of the instrument (Burca et al., 2013; Kockelmann et al., 2015) started in 2008, and construction was finished in 2016. Since then, engineering and scientific commissioning has mainly focused on the neutron imaging capability, although neutron diffraction will eventually be routinely available on the same beamline for samples that can benefit from characterisation using both techniques.

The IMAT instrument utilises neutrons received from the newer Target Station 2 at the ISIS neutron source, which produces short-duration $(<1 \mu \mathrm{s})$ neutron pulses at $10 \mathrm{~Hz}$. These neutrons first pass through a coupled moderator filled with liquid hydrogen cooled to $18 \mathrm{~K}$, which slows them to velocities on the order of $10^{3} \mathrm{~m} \cdot \mathrm{s}^{-1}$ without unduly lengthening the 
these slowed neutrons have a spread of wavelengths comparable to the atomic spacing in ordinary solid matter. Neutrons are transported from the moderator to the IMAT instrument using a supermirror straight neutron guide of $42.8 \mathrm{~m}$ length. The required band of wavelengths is selected using choppers: spinning assemblies that block the neutron path at specific times during their rotation such that only neutrons within a specific range of velocities (and hence wavelengths) are capable of reaching the instrument from the target where the neutrons are produced. IMAT uses three sets of choppers, one for filtering out unwanted high-energy neutrons and gamma radiation, and two for defining the required wavelength band while preventing faster neutrons overtaking slower neutrons from the previous pulse.

In order to create a well-collimated neutron beam suitable for radiography, the neutron beam passes through a pinhole aperture with one of six different selectable diameters $(5,10,20,40,80$ and $100 \mathrm{~mm})$. As the distance from this aperture to the sample position and detector is $\sim 10 \mathrm{~m}$ (the sample typically being close to the imaging plane of the detector), the beam can be assumed to be near-parallel, and the resulting projected image of the sample is not magnified. Accordingly, samples that are smaller than the active area of the detector can be analysed in a single acquisition, while images of larger samples can be assembled piecewise by translating the sample on a motorised stage between successive acquisitions. Rotation of the sample also enables tomographic reconstruction from individual radiographs collected at a series of angles (Anderson et al., 2009). A photograph of the IMAT facility is shown in Figure 1.

For the experiments presented in this paper, neutron radiographs were imaged with an optical camera box (Finocchiaro et al., 2013), with a field-of-view varying from $50 \times 50 \mathrm{~mm}^{2}$ to $200 \times 200 \mathrm{~mm}^{2}$, using a defined continuous wavelength range. This setup detects and records the image by means of a scintillator screen, mirror, lens and a Zyla sCMOS 4.2 Plus 
62 camera (www.andor.com). It should be noted that, IMAT also supports the use of a second

63

64

65

66

67

68

69 detector capable of recording a full neutron spectrum for each of its $55 \mu \mathrm{m}$ pixels, albeit over a smaller $28 \times 28 \mathrm{~mm}^{2}$ field of view (Tremsin et al., 2013).

\section{DATA COLLECTION, PROCESSING AND ANALYSIS}

The basic principle of neutron radiography (similar to X-ray radiography) is that a neutron beam passing through the sample is attenuated. The detector registers the fraction of the initial beam intensity that remains after it has been transmitted by each point in the object. The relationship between the incident intensity $I_{0}$ and the transmitted intensity $I$ is given by the Beer-Lambert law defined as,

$$
I=I_{0} e^{-\mu t}
$$

where $\mu$ is the attenuation coefficient along the path of the neutron through the sample and depends on the selected material and its density and $t$ is the thickness of the sample along this path. For all test measurements presented in this paper, samples were mounted on the tomography stage positioned on the translation/rotation stage system (Figure 1) between the neutron beam flight tube and the detector. The main consideration in selecting the detector for an experiment was its capacity to resolve details of interest with good spatial and temporal resolution. Moreover, for each radiographic setup, the $L / D$ ratio (beam collimation, $L$ being pinhole to sample distance, and $D$ pinhole diameter) was modified based on conditions including the neutron flux, spatial resolution of the detector, and size of the sample. A normalisation procedure was used to eliminate/minimise inhomogeneities of the images obtained that result from the spatial variations in beam intensity, the response of the camera to the beam, or from the electronic noise generated by the camera system. To achieve this, radiographies of the same exposure time were collected without the sample present in the beam (open beam) and with the beam shutter closed (dark field). The image taken with 
87 the sample in the beam (at the same energy as the open beam) was normalised to the open beam after subtracting the dark field from both images. This was done automatically in the software used for reconstruction, or using ImageJ (Rasband et al., 1997).

The reconstruction of the sample projections into a $2 \mathrm{D}$ slice of the object was done using commercial software, e.g. Octopus (Octopus 8.8, inCT, Ghent, Belgium) (https://octopusimaging.eu) or the open-source Python package TomoPy (Argonne National Laboratory; https://tomopy.readthedocs.io/en/latest/). When the reconstruction was completed the volume data obtained was imported into Thermo Scientific ${ }^{\mathrm{TM}}$ Avizo 3D volume rendering software (FEI Visualization Sciences Group; www.fei.com/software/amiraavizo) where the volumetric object was generated and rendered from the stack of image slices.

\section{EXEMPLAR MEASUREMENTS}

We have decided to choose and measure samples of high complexity (in terms of shape, composition, inner structure, dimensions) during the scientific commissioning of IMAT to test the feasibility and the instrument's capabilities for neutron imaging applications in future interdisciplinary life sciences projects.

\subsection{Palaeontology}

Palaeontology is a research field where neutron tomography has significant potential as a non-destructive technique complementing X-ray computed tomography $(\mathrm{CT})$. Over the last decade, it has been used occasionally to study fossil specimens (de Beer et al., 2008; Laaß et al., 2011; Mays et al., 2017). Fossil preservation mechanisms are variable and some specimens have poor X-ray attenuation contrast. This issue is particularly acute, for example, when a fossil and its host rock have similar composition and density: for example the Herefordshire Lagerstätte has calcite $\left(\mathrm{CaCO}_{3}\right)$ fossils within calcium carbonate nodules 
112 (Sutton et al., 2001; Briggs et al., 2012; Briggs et al., 2017). In these cases, X-ray CT 113 provides insufficient detail to fully resolve the preserved fossilized structures (Sutton et al.,

114 2014). To test the suitability of IMAT for studying fossils with poor X-ray attenuation 115 contrast, we applied neutron tomography to fossil crabs from the Eocene of Spain (Figure 2a)

116 that were previously imaged using X-ray CT with limited success (Figure 2b). Imaging at

117 IMAT was carried out using the optical camera box with sCMOS camera (58 $\mu \mathrm{m}$ pixel size; 118 http://doi.org/10.5281/zenodo.825926). The results show that neutron tomography can be 119 successful in probing samples with low X-ray attenuation, capturing previously unseen 120 features from the interior of fossils and making it possible to distinguish between fossil and 121 matrix, even in material with limited internal density contrast (Figure 2c).

122 This pilot study provides an excellent opportunity to introduce scientists from different 123 branches of the palaeontological community to the capabilities of IMAT. Furthermore, they

124 will facilitate a deeper understanding of the strengths and weaknesses of neutron tomography 125 in comparison to the traditional X-ray CT scanning, thus helping the geoscience community 126 target future neutron tomography applications.

\section{4.2. Soil/plants science}

128 One of the essential functions of plant roots is the uptake and transport of water. Due to increasing water restraints on agriculture, there is strong motivation to truly understand plant water uptake. This requires new methods that allow the measurement of water fluxes in roots

131 and the soils that surround them. However, X-rays are not well-suited to characterisation of 132 water distribution, since the contrast ratios between water, plant roots, and other soil constituents are poor. The high neutron attenuation coefficient of hydrogen makes neutron radiography and tomography very attractive techniques for measuring water dynamics in plant-soil systems (Matsushima et al., 2008; Menon et al., 2007; Totzke et al., 2017; Warren 
et al., 2013; Zarebanadkouki et al., 2013) and map the water content distribution in soils

137 (Carminati et al., 2007; Cheng et al., 2012).

On IMAT we propose to develop similar methods to understand water flux in soils,

driven by root activity in order to inform models of water dynamics in agricultural systems.

140 The first step of our soil-plant system research project was to investigate the water

141 distribution into the soil using neutron tomography. A compost specimen was measured

142 because it contained a wide range of elements (organic and inorganic) and could absorb many

143 times its own weight in water, resulting in improved soil water and nutrient availability

144 (Figure 3).

Further tomographic acquisitions will be carried out using different neutron beam and

146 detector parameters, allowing benchmarking of the instrument's performance in elucidating

147 soil water distributions. Moreover, reconstructed neutron data will complement the X-ray CT reconstructions (Keyes et al., 2017; Keyes et al., 2016) in order to validate models describing the dynamics of water transport in the soil region immediately surrounding plant roots.

\subsection{Dentistry application}

151 The first neutron tomography of a human tooth (second molar) on IMAT was acquired using optical camera box equipped with Andor Zyla 4.2 PLUS sCMOS with 2048×2048 pixels, each pixel of $30 \times 30 \mu \mathrm{m}$. A neutron flux density of $10^{7}$ neutrons $/ \mathrm{cm}^{2} / \mathrm{sec}$ at a beam collimation of 250 reached the sample. The neutron tomography scan of the tooth lasted almost 9 hours, and had 1049 projections over $360^{\circ}$ rotation, with 6 flat field and 6 dark field images and 30 second exposure time per image. The Fourier grid reconstruction algorithm from the module tomopy.recon.algorithm (Dowd et al., 1999; Rivers, 2012) was applied for reconstruction, revealing the internal structure of the tooth (pulp chamber, dentin and enamel), which was then virtually extracted and imaged in 3D with Avizo software (Figure 4). These initial measurements allow the quantification of the internal signature of the tooth 
with potential applications including the investigation of endodontic processes and forensic dentistry, or even analysis of bone implant biomaterials if one considers the recent improvements in high-resolution neutron imaging (spatial resolution of $5 \mu \mathrm{m}$ ) (Jakubek et al., 2006; Tremsin et al., 2008).

\section{CONCLUSION}

167 We have shown throughout this paper that IMAT is definitely a reliable neutron imaging tool to be successfully employed in cutting-edge interdisciplinary research of high complexity 169 which bring together experts from different research fields. Because IMAT is a newly built 170 instrument exemplar measurements are of paramount importance since they are an effective 171 means of identifying the best possible matches between the instrument capabilities and 172 applications. The results acquired prove that as well as being complementary to X-ray 173 computed tomography, neutron tomography is also an efficient non-destructive investigation 174 method that can contribute significantly to the progress of life sciences research.

\section{ACKNOWLEDGEMENTS}

The authors are thankful to Dr Luke Clifton (ISIS Biological Sample Specialist) for fruitful discussions and Mrs Asha Lata Dopplapudi (Biology Laboratory Manager) for granting us access to the TS-2 BioLab at the ISIS facility from Rutherford Appleton Laboratory.

Dr Martin Turner acknowledges the support of $\mathrm{CCPi}$, the Collaborative Computational Project in Tomographic Imaging (https://www.ccpi.ac.uk/) funded from EPSRC (grant EP/M022498/1) and CoSeC.

\section{REFERENCES}

1. Anderson, I.S., McGreevy, R.L., Bilheux, H.Z. eds (2009), Neutron Imaging and Applications: A reference for the imaging community, Springer-Verlag US 
2. Benetti, A.R., Jacobsen, J., Lehnhoff, B., Momsen, N.C.R., Okhrimenko, D., Telling, M.T.F., Kardjilov, N., Strobl, M., Seydel, T., Manke, I., Bordallo, H.N. (2015) How mobile are protons in the structure of dental glass ionomer cements?, Scientific Reports, 5: 8972

3. Briggs, D.E., Siveter, D.J., Siveter, D.J., Sutton, M.D., Garwood, R.J., Legg, D. (2012), Silurian horseshoe crab illuminates the evolution of arthropod limbs. Proc. Natl. Acad. Sci. U.S.A, 109, 15702-15705

4. Briggs, D.E., Siveter, D.J., Siveter, D.J., Sutton, M.D., Rahman, I.A. (2017), An edrioasteroid from the Silurian Herefordshire Lagerstätte of England reveals the nature of the water vascular system in an extinct echinoderm. Proc. R. Soc. B, 284, 20171189

5. Burca, G., Kockelmann, W., James, J.A., Fitzpatrick, M.E. (2013), Modelling of an imaging beamline at the ISIS pulsed neutron source, Journal of Instrumentation, 8, P10001

6. Carminati, A. Kaestner, A.,Hassanein, R., Ippisch, O., Vontobel, P., Flühler, H. (2007), Infiltration through series of soil aggregates: Neutron radiography and modelling, Adv. Water Resour. 30, 1168-1178

7. Cheng, C.L., Kang, M., Perfect, E., Voisin, S., Horita, J., Bilheux, H.Z., et al. (2012), Average soil water retention curves measured by neutron radiography, Soil Sci Soc Am J, 76, 1184-1191

8. de Beer, F.C., Prevec, R., Cisneros, J., Abdala, F. (2008), Hidden structure of fossils revealed by neutron and X-ray tomography, Proceedings of the 8th world conference on neutron radiography (WCNR-8), Neutron radiography 8, Destech Publications, Lancaster, $452-461$

9. Dowd, B.A., Campbell, G.H., Marr, R.B., Nagarkar, V.V., Tipnis, S.V., Axe, L, Siddons, D.P. (1999), Developments in synchrotron x-ray computed microtomography at the national synchrotron light source, Proc. SPIE, volume 3772, 224-236 
10. Finocchiaro, V., Aliotta, F., Tresoldi, D., Ponterio, R.C., Vasi, C.S., Salvato, G. (2013), The autofocusing system of the IMAT neutron camera, Rev. Sci. Instrum., 84, 093701

11. Heinz Maier-Leibnitz Zentrum et al. (2015) ANTARES: Cold neutron radiography and tomography facility, Journal of large-scale research facilities, 1, A17

12. Jakubek, J., Granja, C., Holy, T., Lehmann, E., Linhart, V., Pospisil, S., Rypar, V., Uher, J., Vacik, J., Vavrik, D., Vykydal, Z., Cevallos, M. (2006) Neutron imaging and tomography with Medipix2 and dental micro-roentgenography, Nuclear Instruments and Methods in Physics Research A 569, 205-209

13. Kaestner, A., Hartmann, S., Kuhne, G. et al. (2011), The ICON beamline - A facility for cold neutron imaging at SINQ, Nuclear Instruments and Methods in Physics Research A, 659 (1), 387-393

14. Kardjilov, N., Hilger, A., Manke, I., Woracek, R., Banhart, J. (2015), CONRAD-2: the new neutron imaging instrument at the Helmholtz-Zentrum Berlin, Journal of Applied Crystallography, 49, 195-202

15. Keyes, S., Zygalakis, K., \& Roose, T. (2017), Explicit structural modelling of root-hair and soil interactions at the micron-scale parameterized by synchrotron X-ray computed tomography, Bulletin of Mathematical Biology, 79 (12), 2785-2813

16. Keyes, S., Gillard, F., Soper, N., Mavrogordato, M. N., Sinclair, I., \& Roose, T. (2016), Mapping soil deformation around plant roots using in vivo 4D X-ray computed tomography and digital volume correlation, Journal of Biomechanics, 49 (9), 1802-1811

17. Kiyanagi, Y., Shinohara, T., Kai, T., Kamiyama, T. Sato, H. et al. (2013), Present status of research on pulsed neutron imaging in Japan, Physics Procedia, 43, 92-99 (2013)

18. Kockelmann, W., Burca, G., Kelleher, J.F., Kabra, S. et al (2015), Status of the neutron imaging and diffraction instrument IMAT, Physics Procedia, 69, 71 - 78 
19. Laaß, M., Hampe, O., Schudack, M., Hoff, C., Kardjilov, N. and Hilger, A. (2011), New insights into the respiration and metabolic physiology of Lystrosaurus, Acta Zoologica, 92, $363-371$

20. Lehmann, E., Vontobel, P., Wiezel, L. (2001), Properties of the radiography facility NEUTRA at SINQ and its potential for use as European reference facility, Nondestr. Test Eval. 16, 191-202

21. Mays, C., Bevitt, J. J., Stilwell, J. D. (2017), Pushing the limits of neutron tomography in palaeontology: Three dimensional modelling of in situ resin within fossil plants, Palaeontologia Electronica, 20 (3), 1-12

22. Matsushima, U., Kardjilov, N., Hilger, A., Graf, W., Herppich., WB. (2008), Application potential of cold neutron radiography in plant science research, J Appl Bot Food Qual, 82, $90-98$

23. Menon, M., Robinson, B., Oswald, S. E., Kaestner, A., Abbaspour, K. C., Lehmann, E. and Schulin, R. (2007), Visualization of root growth in heterogeneously contaminated soil using neutron radiography, European Journal of Soil Science, 58, 802-810

24. Rasband, W.S., ImageJ, U. S. National Institutes of Health, Bethesda, Maryland, USA, https://imagej.nih.gov/ij/, 1997-2016

25. Rivers, M.L., (2012), Tomorecon: high-speed tomography reconstruction on workstations using multi-threading, Proc. SPIE, volume 8506, 85060U-85060U-13

26. Santodonato, L., Bilheux, H., Bilheux, J. et al. (2015), The CG-1D Neutron Imaging Beamline at the Oak Ridge National Laboratory High Flux Isotope Reactor, Physics Procedia, 69, 104-108

27. Sutton, M.D., Briggs, D.E., Siveter, D.J., Siveter, D.J. (2001), Methodologies for the visualization and reconstruction of three-dimensional fossils from the Silurian Herefordshire Lagerstätte. Palaeontol. Electron., 4, 1-17 
28. Sutton, M., Rahman, I.A., Garwood, R. (2014), Techniques for Virtual Palaeontology, John Wiley \& Sons

29. Teixeira, S.C.M., Ankner, J., Bellissent-Funel, M.C., Bewley, R. et al. (2008), New sources and instrumentation for neutrons in biology, Chem Phys., 345 (2-3), 133-151

30. Tötzke, C., Kardjilov, N., Manke, I., Oswald, S.E. (2017), Capturing 3D Water Flow in Rooted Soil by Ultra-fast Neutron Tomography, Scientific Reports, 7

31. Tremsin, A.S., Vallerga, J.V., McPhate, J.B., et al. (2013), High resolution photon counting with MCP-Timepix quad parallel readout operating at $>1 \mathrm{KHz}$ frame rates, IEEE Trans. Nucl. Sci., 60 (2), 578-585

32. Tremsin, A.S., Vallerga, J.V., McPhate, J.B., Siegmund, O.H.W., Feller, W.B., Crow, L., Cooper, R.G. (2008), On the possibility to image thermal and cold neutron with sub-15 mm spatial resolution, Nuclear Instruments and Methods in Physics Research A, 592, 374-384

33. Weisman, M.I., Brown, M. (1971), Neutron radiography of the dental pulp: A preliminary report, Oral Surgery, Oral Medicine, Oral Pathology, 32 (3), 487-492

34. Warren, J.M., Bilheux, H., Kang, M., Voisin, S., Cheng, C.L., Horita, J., Perfect, E. (2013), Neutron imaging reveals internal plant water dynamics, Plant Soil, 366 (1-2), 683693

35. Yunus, S.O., Oyewumi, K.J., Amosa, M.K. (2010), Analytical Methods Using Instrumental Neutron Activation Analysis: Determining the Radionuclides, Concentrations and Differential Factors of Biological Samples, VDM Verlag Dr. Müller

36. Zarebanadkouki, M., Kim, Y. X., Carminati, A. (2013), Where do roots take up water? Neutron radiography of water flow into the roots of transpiring plants growing in soil, New Phytol., 199, 1034-1044 www.isis.stfc.ac.uk/ (accessed 01/02/2018) https://octopusimaging.eu/ (accessed 25/01/2018) 
https://tomopy.readthedocs.io/en/latest/ (accessed 24/01/2018)

https://www.fei.com/software/amira-avizo/ (accessed 28/01/2018) 
Figure 1. The IMAT beamline: neutron imaging setup with the optical camera box mounted on the robotic arm

Figure 2. (a) Fossil crabs from the Eocene (40 million years old) of Spain; (b) slice through reconstructed X-ray CT (scanned on a Nikon Metrology HMX ST 225 system at the Natural History Museum, London, using a $1.0 \mathrm{~mm}$ thick Copper filter, $225 \mathrm{kV}$ voltage and $180 \mu \mathrm{A}$ current, giving a tomographic dataset with a voxel size of $46 \mu \mathrm{m})$ of fossil crabs; (c) slice through reconstructed neutron tomography of fossil crabs on IMAT.

Figure 3. (a) Photograph of the NT experimental setup of the quartz glass tube (boron free) filled with compost, $10 \mathrm{~mm}$ in diameter. Radiographies were recorded with Zyla sCMOS 4.2 Plus camera (30 $\mu m$ pixel size) and computed to a $3 D$ volume stack (b) The attenuation profile of the neutron data shows that the water is predominantly held in the clay aggregates, rather than in the hard mineral grains. The soil texture (poorly described by NT) will be complemented by the future synchrotron X-rays $C T$.

Figure 4. (a) Photograph of human tooth; (b) Neutron radiograph of tooth; (c-e) Tomographic $3 D$ reconstruction of tooth with 3 segmented areas showing (pulp chamber, dentin and enamel) 
Figure 1

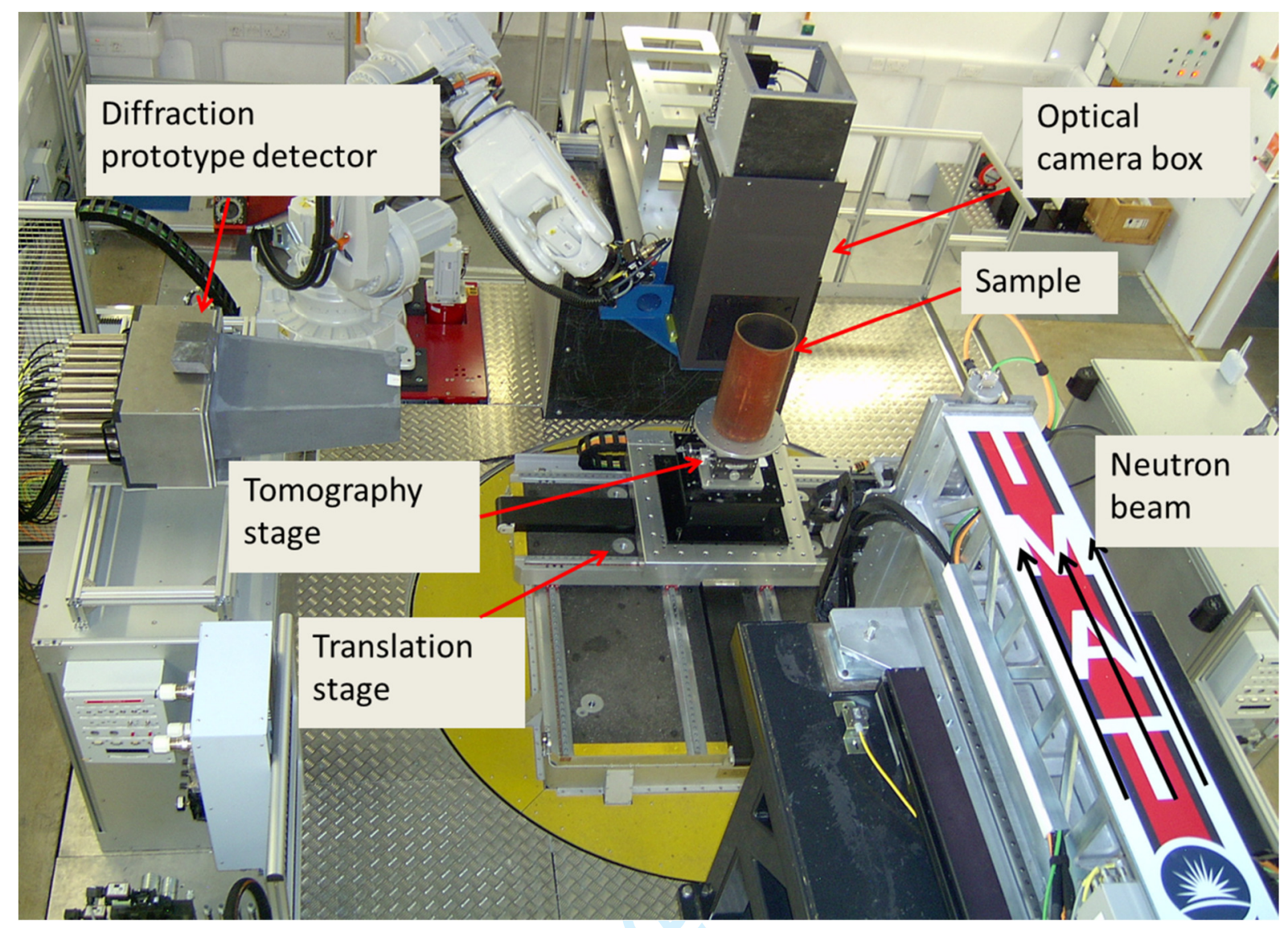

Figure 2

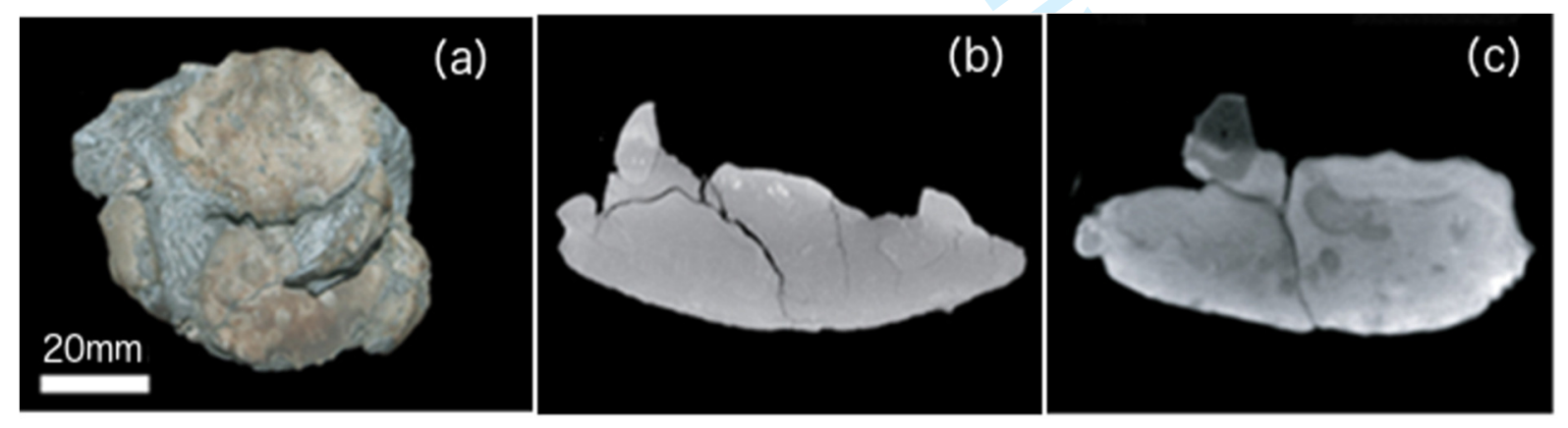


Figure 3
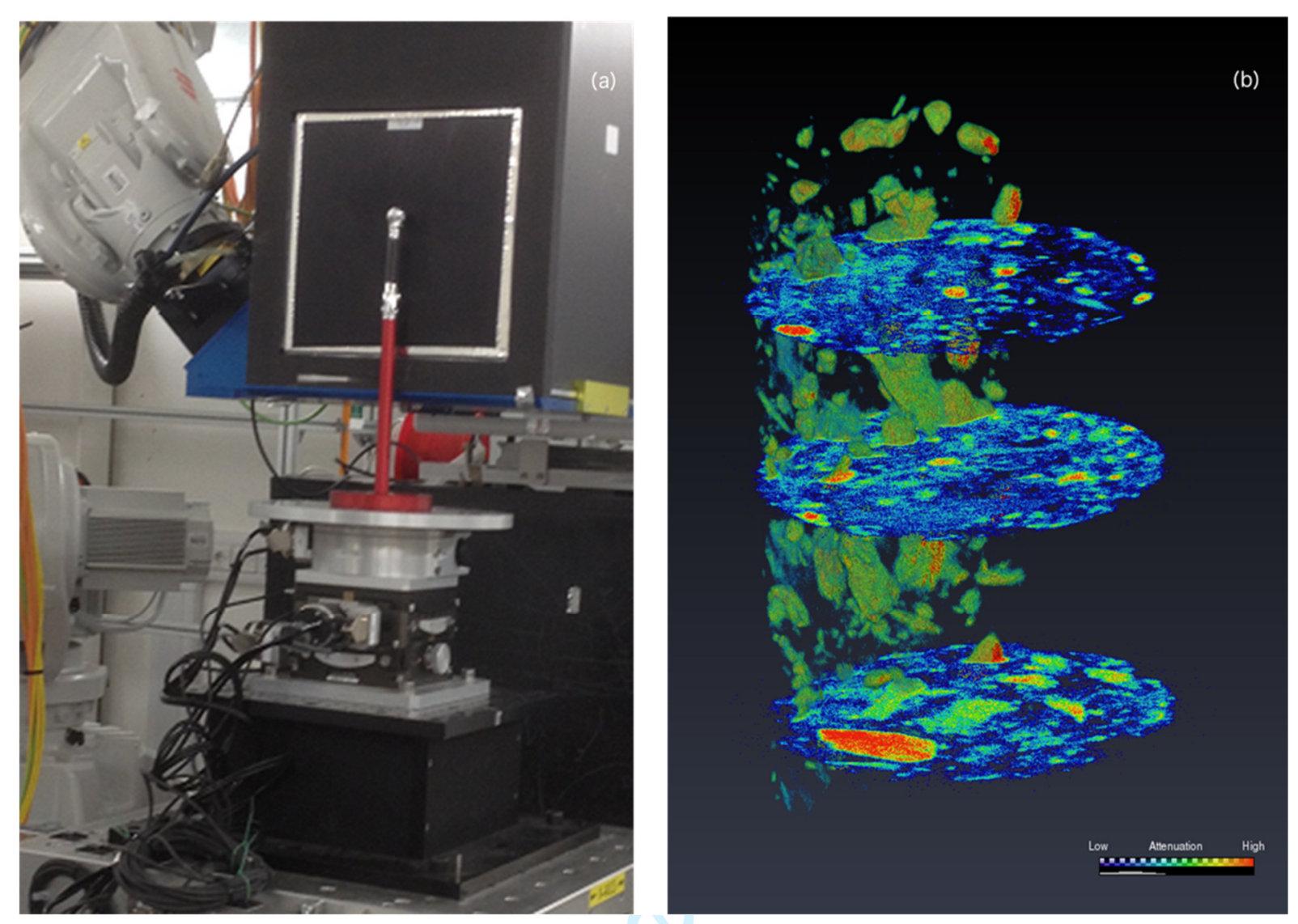

Figure 4

(a)

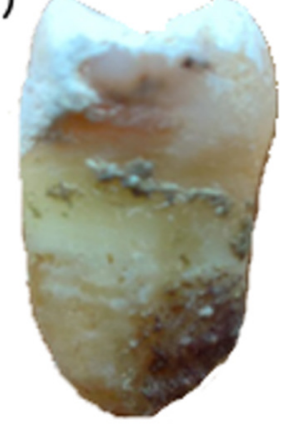

(d)

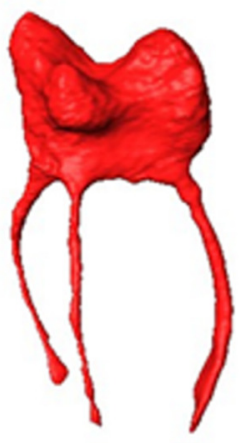

(b)

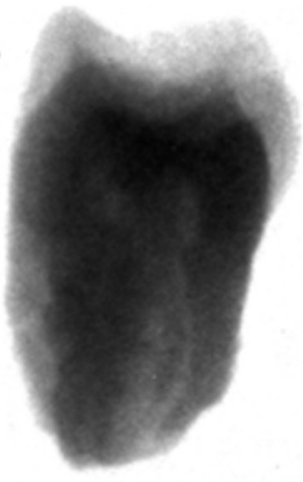

(e)

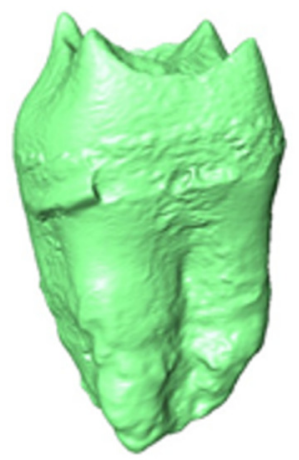

(c)

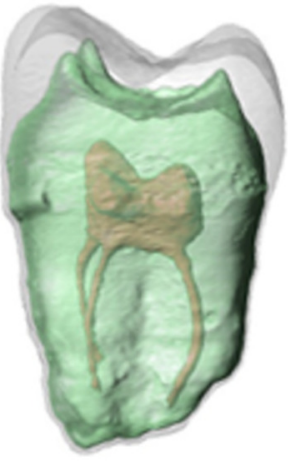

(f)

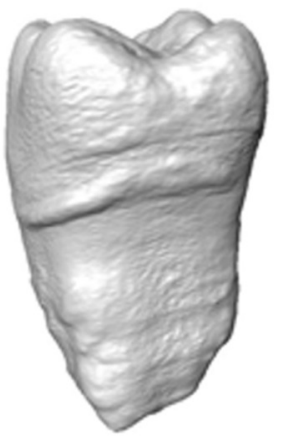




\section{Lay description}

Neutrons form a highly penetrating radiation passing through matter without damaging or structurally modifying it, a property that makes them the ideal tool for many kinds of complementary material investigations. Moreover, the strong interaction of neutrons with hydrogen and their ability to distinguish between hydrogen and deuterium with no radiation damage make neutrons a good probe for imaging biological specimens. The recent technological developments of sources and detectors improved the capabilities of neutron imaging instruments and also have facilitated the use of neutron imaging on a much wider scale than before. Neutron imaging is proving its advantages as being complementary to other known methods of investigation such as X-ray imaging or magnetic resonance imaging and it is no surprise that it is not only employed in engineering or archeaology, but also in life sciences. This definitely opens new perspectives for a more interdisciplinary approach in contemporary science. Within the last decade a neutron imaging beamline, IMAT, was designed and built at the ISIS Neutron and Muon Source, UK, to meet the increasing demands of researchers from different fields, spanning from materials engineering to biology. The results presented here, acquired from first measurements on different biological samples during the scientific commissioning of IMAT beamline show the instrument capability and its suitability to palaeontology, agriculture (soil-plants systems) or dentistry applications. 